\title{
Evaluación de la capacidad de diseño de filtro de capacidades conmutadas con Particle Swarm Optimization (PSO)
}

\section{Evaluation of design capability of switched capacitor filter with Particle Swarm Optimization}

Presentación: 6-7/10/2020

\section{Doctorando:}

\section{Gonzalo Tomás Vodanovic}

Grupo de Estudio en Calidad Mecatrónica, Facultad regional Villa María, Universidad Tecnológica Nacional, Argentina. Grupo de Desarrollo Electrónico e Instrumental, Facultad de Matemática, Astronomía y Física, Universidad Nacional de Córdoba, Argentina. gvodanovic@unc.edu.ar

\section{Director:}

\section{Eduardo Romero}

\author{
Co-directora:
}

\section{Gabriela Peretti}

\section{Resumen}

En el presente trabajo, se analiza la capacidad del algoritmo Optimización por Enjambre de Partículas para diseñar filtros analógicos de capacidades conmutadas embebidos en una plataforma deseñales mixtas para una amplia variedad de especificaciones. Se utiliza la tasa de convergencia para medir el rendimiento del optimizador en estos distintos escenarios y se analizan las razones por la que esta métrica varía para distintas especificaciones de filtro.

Palabras clave: Optimización por enjambre de partículas, filtro analógico, capacidades conmutadas, PSoC1

\begin{abstract}
In the present work, we analyze the ability of the Particle Swarm Optimization algorithm to design switched capacities analog filters embedded in a particular mixed signal platform for a wide variety of specifications. The convergence rate is used to measure the performance of the optimizer in these different scenarios, and are discussed the reasons this metric varies for different filter specifications.
\end{abstract}

Keywords: Particle Swarm Optimization, analog filter, switched capacities, PSoC1.

\section{Introducción}

El optimizador por enjambre de partículas (PSO, Particle Swarm Optimization) es un método estocástico de optimización inspirado en el comportamiento social de animales que viven en grupos (Eberhart y Kennedy, 1995). El comportamiento (velocidad y posición) de cada individuo (partícula) es el resultado de una combinación de influencias cognitivas, sociales y estocásticas. La función de fitness es una medida de la calidad de una partícula como resultado. En cada iteración, el algoritmo computa esta función para diferentes puntos del espacio de búsqueda multidimensional, utilizando un conjunto de partículas. Luego determina nuevas posiciones a explorar a partir de su conocimiento individual y del colectivo. Esto se repite hasta alcanzar el número máximo de iteraciones o hasta encontrar una solución adecuada. 
En el presente trabajo se aplica PSO para efectuar el diseño de filtros pasa bajo de capacidades conmutadas. Se ha adoptado como estructura a la disponible en la plataforma PSoC 1 de Cypress Semiconductor (Murphy, 2012) (Ess, 2012), dadas las posibilidades de obtener resultados experimentales a futuro. Las características generales de la plataforma utilizada y la topología de los filtros han sido previamente descritas en Vodanovic y col., 2019.

Para este problema en particular, la función de fitness es una suma ponderada de los errores entre las especificaciones calculadas $\left[\mathrm{f}_{0}, \mathrm{~d}, \mathrm{G}\right]$ y los valores deseados $\left[\operatorname{espec}\left(f_{0}\right), \operatorname{espec}(d), \operatorname{espec}(G)\right]$, como puede verse en la ecuación 1.

$$
\text { fitness }=\frac{f_{0}-\operatorname{espec}\left(f_{0}\right)}{\operatorname{espec}\left(f_{0}\right)}+\frac{d-\operatorname{espec}(d)}{\operatorname{espec}(d)}+\frac{G-\operatorname{espec}(G)}{\operatorname{espec}(G)}
$$

Para cada partícula $K=\left[C_{1}, C_{2}, C_{3}, C_{4}, C_{A}, C_{B}\right]$, se calculan frecuencia de corte $\left(f_{0}\right)$, ganancia $(G)$ y factor de amortiguamiento (d) con las ecuaciones 2 a 4 (Ess, 2012).

$$
f_{0}=\frac{f_{s}}{2 \pi} \frac{\sqrt{C_{2} C_{3}}}{\sqrt{C_{A} C_{B}-\frac{1}{2} C_{4} C_{3}+\frac{1}{4} C_{2} C_{3}}} \quad \text { (2) } \quad G=-\frac{C_{1}}{C_{2}} \quad \text { (3) } \quad d=\frac{C_{4}}{\sqrt{C_{A} C_{B}-\frac{1}{2} C_{4} C_{3}+\frac{1}{4} C_{2} C_{3}}}
$$

Cuando el error en cada una de las especificaciones es menor al 5\%, se considera a la partícula como una solución y el proceso de optimización se finaliza. Existen diversas implementaciones de este optimizador, el presente trabajo utiliza la versión estandarizada en 2011 (Zambrano-Bigiarini y col., 2013) denominada SPSO2011 (Clerc, 2020).

Se plantea una evaluación intensiva del algoritmo, sobre un amplio abanico de posibilidades de diseño, diferente a las planteadas previamente por otros autores (Náhlıék y col., 2015) (Vural y col., 2011), en las cuales el desempeño de los algoritmos se evalúa en pocos casos de estudio. La exploración efectuada se orienta a la determinación de la habilidad del método para resolver los casos presentados. Los resultados obtenidos en este trabajo son promisorios y permiten visualizar una aplicación del mismo en futuros trabajos.

\section{Configuración del experimento}

Con el objetivo de analizar la capacidad del algoritmo para diseñar filtros de capacidades conmutadas, se selecciona un amplio rango de especificaciones, obtenidas mediante la combinación de distintos valores para los tres parámetros principales. Para la ganancia se eligen $[0,5 ; 1,0 ; 2,0]$, para la frecuencia de corte se utilizan 200 valores en el rango entre $20 \mathrm{~Hz}$ y $100 \mathrm{Khz}$ con distribución logarítmica y para el factor de amortiguamiento se seleccionaron $[0,25 ; 1,0 ; 2,0]$. Esto conduce a 1800 filtros diferentes a diseñar. Dado que el espacio de búsqueda es relativamente pequeño, se pudo determinar que existen 17 casos que no tienen al menos una solución sintetizable en el PSoC1 (Ess, 2012). Con el propósito de evaluar el comportamiento del algoritmo, se lo ejecuta 100 veces para cada filtro, modificando la inicialización de la población inicial. Esto brinda un panorama más amplio sobre la convergencia de PSO. Los parámetros del optimizador se eligen de acuerdo al estándar 2011 (Zambrano-Bigiarini y col., 2013) y, a la experiencia obtenida a partir de distintas corridas: tamaño de enjambre: 35; peso inercial: 0,8; Aceleración Cognitiva: 1,62; Aceleración Social: 1,62. Se selecciona un máximo de 1000 iteraciones dado que el objetivo del presente trabajo, es analizar la variación de la tasa de convergencia sin que este parámetro sea una limitación. Los parámetros se mantienen fijos para todos los diseños planteados. Esto obedece a la necesidad de evaluar el método como herramienta de diseño general, sin adaptaciones por parte del usuario.

\section{Resultados obtenidos}

En la figura 1 puede verse la distribución del número de iteraciones que el optimizador necesita para encontrar una solución, para los 1783 problemas de diseño planteados (filtros con diferentes especificaciones). Los datos presentados en las figuras tienen en cuenta todos los filtros y todas las corridas de PSO. De las 178,300 ejecuciones del algoritmo, en 169,385 casos se obtuvo una solución, por lo tanto, en el 95\% de los casos el algoritmo converge. Como se muestra en el gráfico, la media de iteraciones obtenida es de 75. 


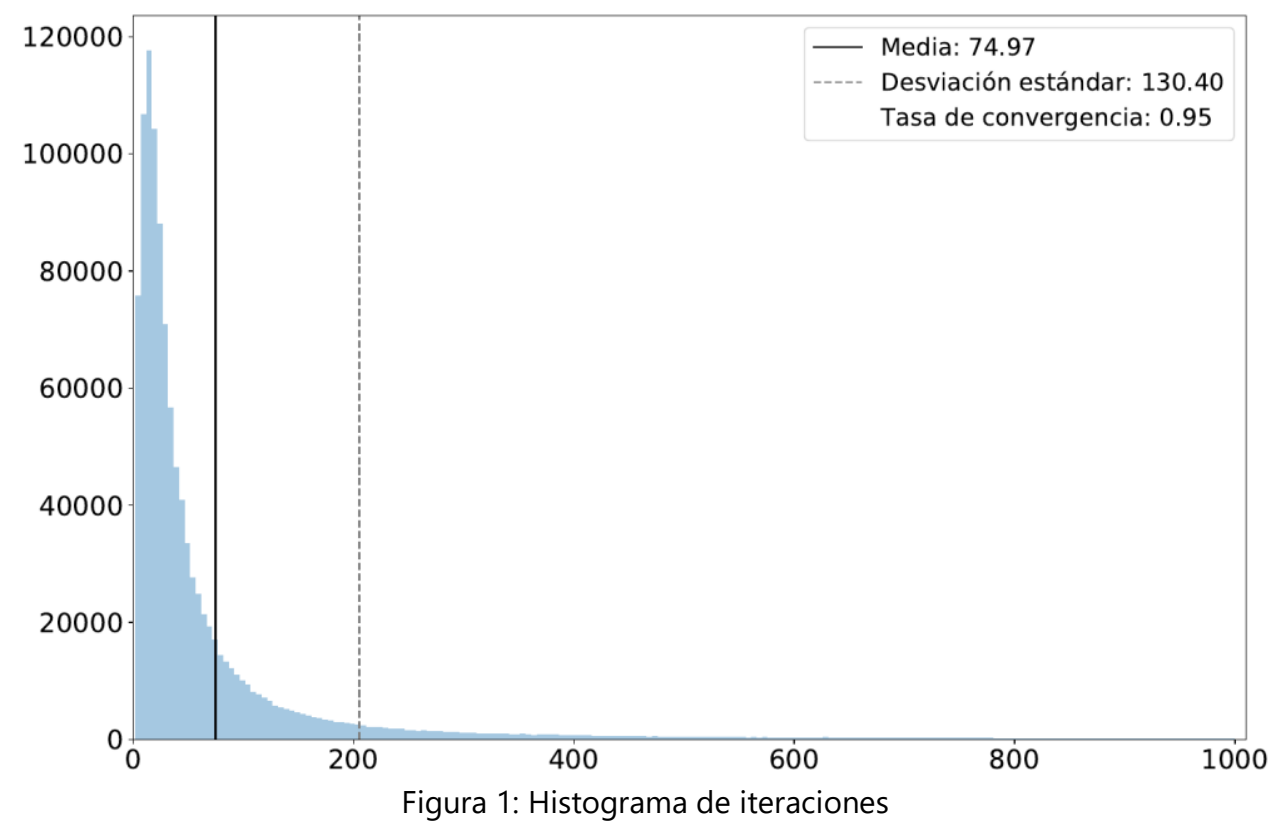

La tasa de éxito o convergencia (Trelea, 2003) se calcula como el número de veces que el PSO converge dividido la cantidad de corridas que se realizaron. El gráfico de la figura 2 muestra la tasa de convergencia respecto a una lista ordenada de números que identifican a cada filtro. Existen casos donde la tasa de convergencia es cero debido a que se incluye los casos que no tienen soluciones (puntos en naranja). En todos los casos con solución (puntos en azul), el algoritmo converge al menos dos veces de las 100 corridas.

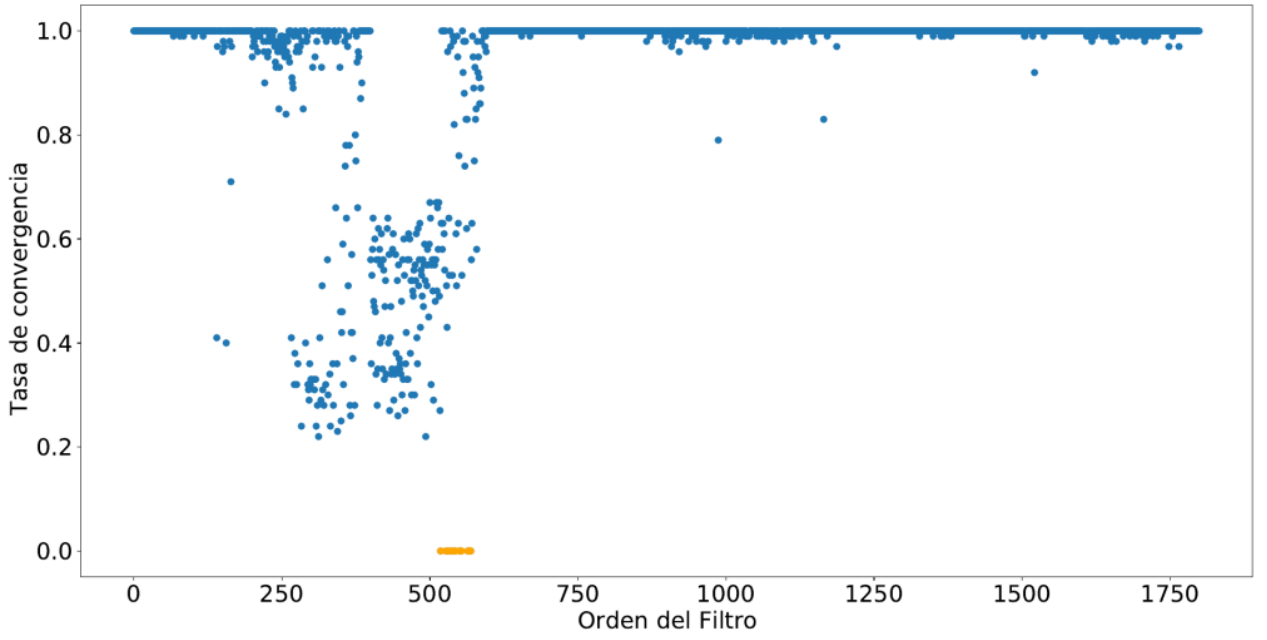

Figura 2: Tasa de convergencia respecto al orden de filtro

Es necesario destacar la existencia de una región con baja tasa de convergencia para los órdenes entre 250 y 600 aproximadamente, lo cual coincide con los filtros donde se combina ganancia igual a $0,5 \mathrm{y}$ factor de amortiguamiento superior a 0,25. Debido a esto, se realiza un análisis de cómo afecta cada una de las especificaciones del filtro a la tasa de convergencia. En las figuras 3a, 3 b y $3 c$ se muestra la tasa de convergencia respecto a la frecuencia de corte, la ganancia y el factor de amortiguamiento, respectivamente. Al calcular la tasa de convergencia para estos tres gráficos se consideran todas las corridas. En cada uno de los gráficos hay dos curvas, en una se consideraron los 1800 casos (en color naranja) y otra donde solo se consideraron los casos que cuentan con al menos una solución (en azul).

En el caso de la frecuencia (figura 3a), la tasa de convergencia se mantiene relativamente constante, aunque para frecuencias mayores a $1 \mathrm{KHz}$, existe una mayor variabilidad. En el caso de ganancia, (figura $3 \mathrm{~b}$ ) se observa que cuando esta es mayor o igual a 1, la tasa de convergencia es cercana a 1 (considerando los casos con al menos una solución). Finalmente, puede verse que, para mayores valores del factor de amortiguamiento, la tasa de convergencia disminuye. A partir de estos gráficos, es posible identificar los casos con ganancia de $0,5 \mathrm{y}$ factor de amortiguamiento de 2,0 como aquellos de mayor dificultad para el optimizador. 


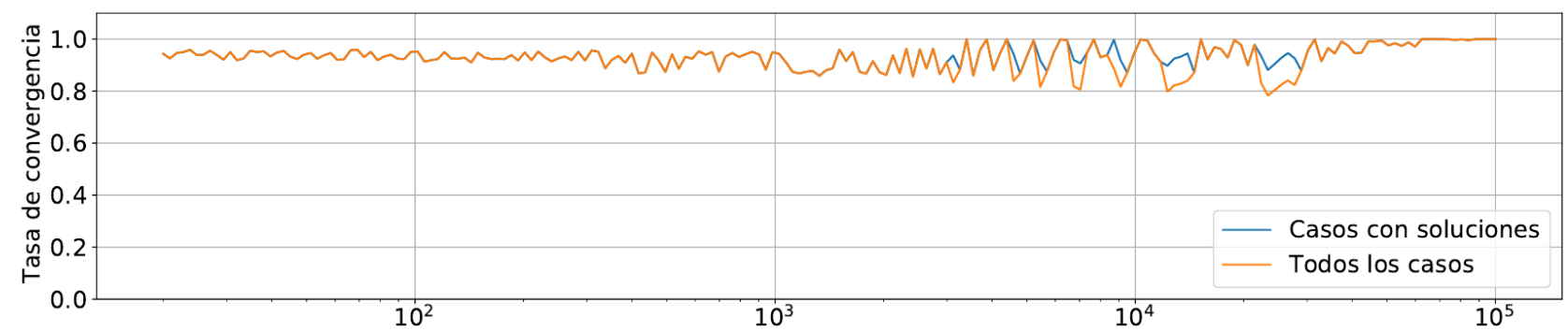

(a) Tasa de convergencia respecto a $\mathrm{f}_{0}$

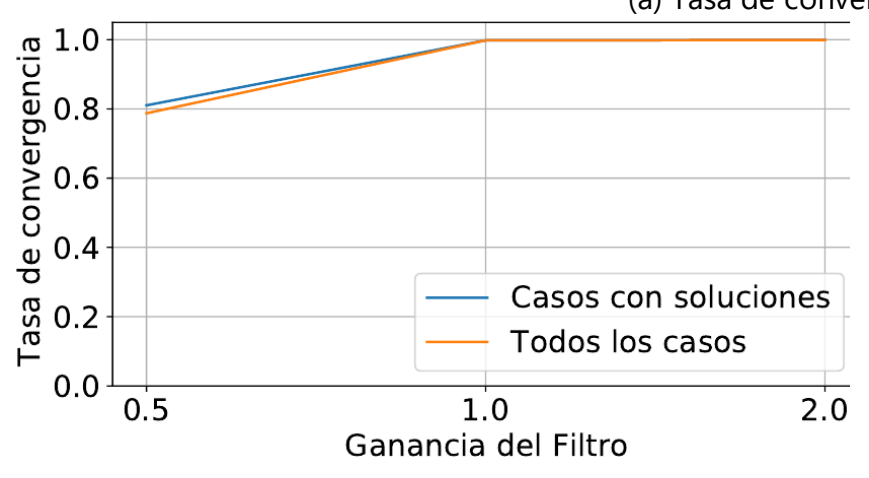

(b) Tasa de convergencia respecto a $G$

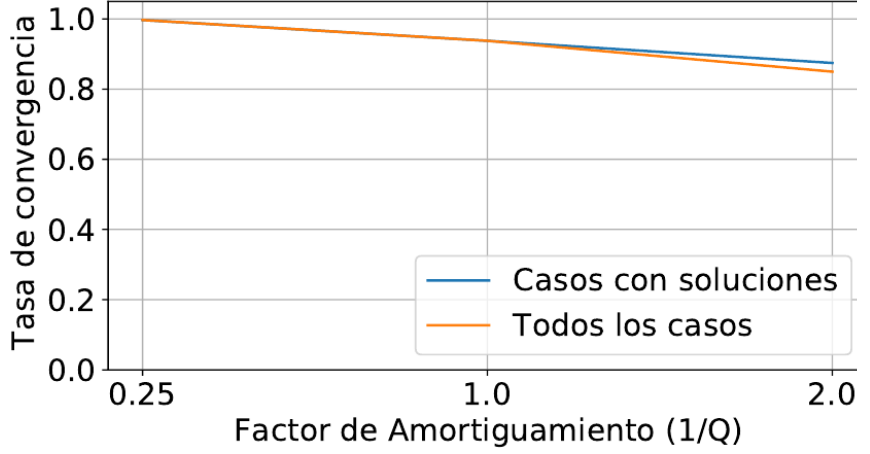

(c) Tasa de convergencia respecto a $\mathrm{d}$

Figura 3: Tasa de convergencia respecto a $f_{0}, d$ y $G$

Con el objeto de analizar los errores de las soluciones obtenidas por PSO respecto de las especificaciones deseadas, se presentan los histogramas de las figuras $4 \mathrm{a}$ y $4 \mathrm{~b}$ (error para frecuencia y para factor de amortiguamiento respectivamente). Como puede verse, en el $90 \%$ de los casos, se logra una solución con una precisión menor al 2,2\% para el factor de amortiguamiento y menor al 3\% respecto de la frecuencia de corte.

El histograma del error respecto a la ganancia no se muestra debido a que el error es siempre $0 \%$. Esto se debe a que, como puede verse en la ecuación 3 , la ganancia depende de los capacitores $C_{1}$ y $C_{2}$, sin embargo, el primero no es utilizado para calcular ninguna otra especificación de filtro (ecuaciones 2 y 4) y, por lo tanto, este capacitor no es elegido por el PSO, sino que se calcula como $\mathrm{C}_{1}=\mathrm{G} \times \mathrm{C}_{2}$.

\section{Análisis de convergencia}

Se destaca que cada uno de los filtros tomados como casos, se constituye en un escenario de diseño diferente para el optimizador. Esto se debe a que la cantidad de soluciones posibles y la distancia entre ellas varía de un caso a otro debido a los valores de capacitores disponibles en el PSoC1. Dado que el espacio de búsqueda es relativamente pequeño (4194304 combinaciones posibles de capacitores), es factible determinar las soluciones y sus distancias. Una mayor cantidad de soluciones brindaría al algoritmo mayores posibilidades de convergencia, las cuales se verían comprometidas si la distancia entre ellas es pequeña. Una mayor dispersión de las soluciones en el espacio de búsqueda, favorece la exploración dado que aumenta la probabilidad de que una partícula inicialice en una posición cercana a una solución y, por lo tanto, deba trasladarse menos por el espacio de búsqueda. A esto se le suma, que en la etapa de explotación, la existencia de varias de ellas en la cercanía puede ser contraproducente para el movimiento de la partícula. 


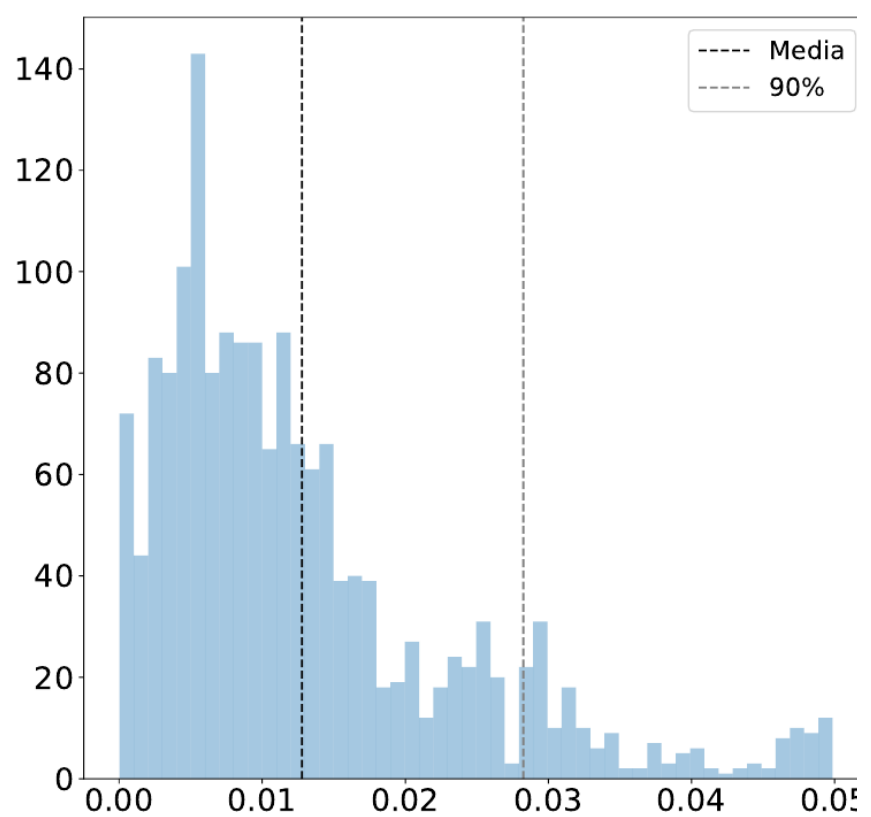

(b) Tasa de convergencia respecto a $\mathrm{G}$

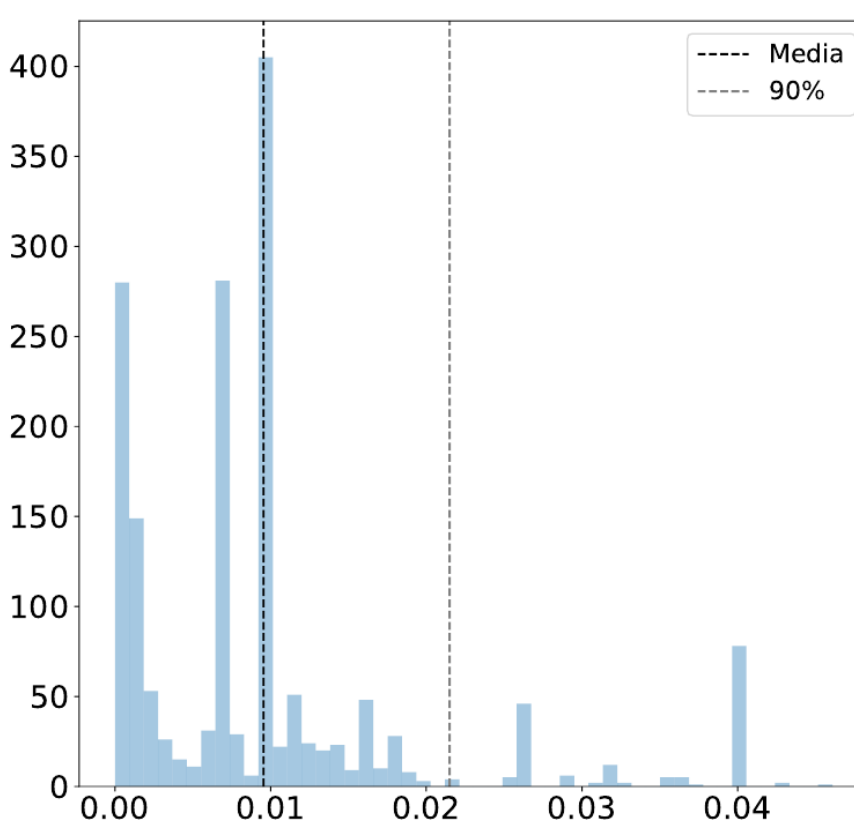

(c) Tasa de convergencia respecto a d

Figura 4: Histogramas de error

El gráfico de la figura 5 muestra el promedio de la cantidad de soluciones (curva en azul) y de la distancia euclidiana entre ellas (curva naranja) para cada valor de frecuencia de corte. Como puede verse, ambas métricas presentan oscilaciones en sus valores, pero con un valor medio que se mantiene razonablemente constante hasta aproximadamente $50 \mathrm{KHz}$. Por encima de esa frecuencia aumenta la cantidad de soluciones, pero también la distancia entre las mismas. Como se dijo, estos valores altos favorecen la convergencia, justificando que se aproxime a la unidad. Cabe destacar que no se observa un cambio muy grande en la tasa de convergencia debido a que ya está en valores altos por encima de $50 \mathrm{KHz}$.

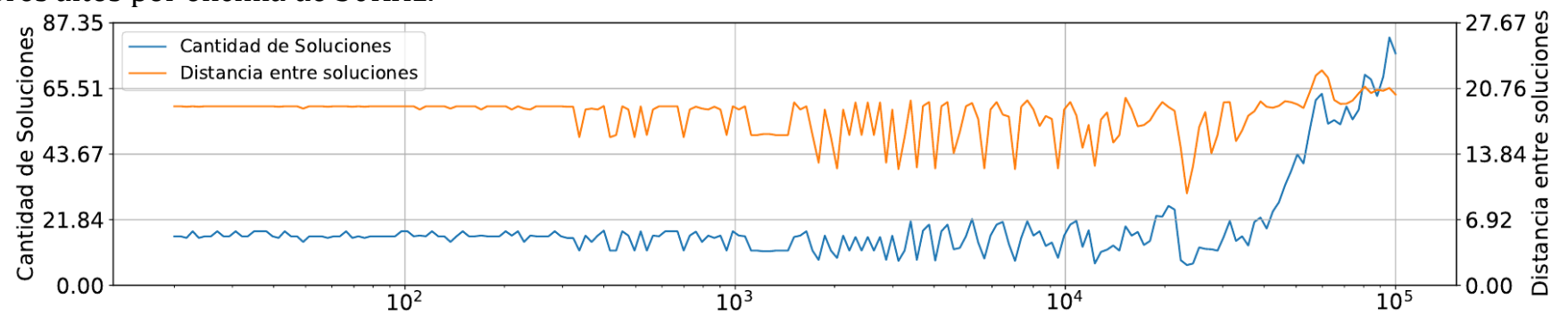

Figura 5: Número de soluciones (promedio) y distancia (promedio) entre ellas respecto a la frecuencia de corte

En la tabla 1 se muestran el promedio del número de soluciones y de la distancia entre ellas para tres valores de amortiguamiento y ganancia. Cada uno de estos valores se obtienen considerando todos los casos que cuentan con una determinada ganancia o factor de amortiguamiento en particular. Como puede verse en la figura 3b, la tasa de convergencia disminuye cuando la ganancia es 0,5 . Esto puede atribuirse a una disminución de las soluciones y a una menor distancia entre las mismas. Por otro lado, como puede verse en el gráfico de la figura 3c la tasa de convergencia baja cuando el factor de amortiguamiento supera a 0,25. La cantidad de soluciones se mantiene constante hasta un factor de amortiguamiento de 1, pero a partir de 0,25 comienza a disminuir la distancia entre las mismas. Se atribuye la disminución de la tasa de convergencia a esta baja en la distancia. Superado el valor 1 se hacen notar la reducción tanto la cantidad de soluciones como la distancia entre las mismas.

\begin{tabular}{|c|c|c|c|c|c|c|c|}
\hline $\mathbf{d}$ & $\begin{array}{c}\text { Tasa de } \\
\text { Convergencia }\end{array}$ & $\begin{array}{c}\text { Número de } \\
\text { Soluciones }\end{array}$ & $\begin{array}{c}\text { Distancia entre } \\
\text { las soluciones }\end{array}$ & $\mathbf{G}$ & $\begin{array}{c}\text { Tasa de } \\
\text { Convergencia }\end{array}$ & $\begin{array}{c}\text { Número de } \\
\text { Soluciones }\end{array}$ & $\begin{array}{c}\text { Distancia entre } \\
\text { las soluciones }\end{array}$ \\
\hline 0,25 & 0,99 & 23,76 & 20,72 & 0,5 & 0,81 & 12,29 & 12,59 \\
\hline 1 & 0,93 & 23,93 & 19,15 & 1 & 0,99 & 19,15 & 15,35 \\
\hline 2 & 0,87 & 19,6 & 16,5 & 2 & 0,99 & 19,59 & 16,49 \\
\hline
\end{tabular}

Tabla 1: Número de soluciones (promedio) y distancia (promedio) entre ellas para G y d 


\section{Conclusión}

Diseñar filtros de diversas especificaciones mediante el optimizador por enjambre de partículas sin adaptar los parámetros implica, para el algoritmo, un desafío distinto en cada caso. Se utilizó la tasa de convergencia para medir el desempeño del PSO en cada escenario planteado. Luego de evaluar 1800 filtros distintos, se obtuvo una tasa de convergencia del $95 \%$ y en el $90 \%$ de los casos, se diseñaron filtros con un error menor al 3\% en las tres especificaciones. Por otro lado, pudo determinarse que, para todas las frecuencias de corte evaluadas, la tasa de convergencia se mantuvo relativamente constante. Sin embargo, para ganancias inferiores a 1 y factores de amortiguamiento superiores a 1, la tasa de convergencia disminuye. También se demostró que esto ocurre debido a que para estos casos existen menos soluciones o se encuentran a menor distancia euclidiana entre ellas.

\section{Referencias}

Clerc, M. (2020). Particle Swarm Central. Recuperado desde http://www.particleswarm.info/. (En línea. ultimo acceso - agosto 2020)

Eberhart, R. \& Kennedy, J. (1995). A new optimizer using particle swarm theory. En MHS'95. Proceedings of the Sixth International Symposium on Micro Machine and Human Science (pp. 39-43). Ieee.

Ess, V. (2012). Understanding PSoC R 1 Switched Capacitor Analog Blocks.

Murphy, R. (2012). Getting Started with PSoC 1. Cypress Semiconductor.

Náhliék, J., Hospodka, J., Šubrt, O. \& Chávez, A. B. (2015). Optimization methods for switched capacitor circuits. En 2015 International Conference on Applied Electronics (AE) (pp. 165-168). IEEE.

Trelea, I. C. (2003). The particle swarm optimization algorithm: convergence analysis and parameter selection. Information processing letters, 85(6), 317-325.

Vodanovic, G. T., Romero, E. \& Peretti, G. (2019). Diseño de filtros analógicos de capacidades conmutadas con Optimización por Enjambre de partıéculas. A JEA, (4).

Vural, R. A., Yildirim, T., Kadioglu, T. \& Basargan, A. (2011). Performance evaluation of evolutionary algorithms for optimal filter design. IEEE transactions on evolutionary computation, 16(1), 135-147.

Zambrano-Bigiarini, M., Clerc, M. \& Rojas, R. (2013). Standard particle swarm optimisation 2011 at cec-2013: A baseline for future pso improvements. En 2013 IEEE Congress on Evolutionary Computation (pp. 23372344). IEEE. 